\title{
Thermal ablation in non-small cell lung cancer: a review of treatment modalities and the evidence for combination with immune checkpoint inhibitors
}

\author{
Kanishka Rangamuwa ${ }^{1,2}$, Tracy Leong ${ }^{3}$, Clare Weeden ${ }^{4}$, Marie-Liesse Asselin-Labat ${ }^{4}$, Steven Bozinovski ${ }^{5}$, \\ Michael Christie ${ }^{6}$, Tom John ${ }^{7}$, Phillip Antippa ${ }^{8}$, Louis Irving ${ }^{1}$, Daniel Steinfort ${ }^{1,2}$ \\ ${ }^{1}$ Department of Respiratory Medicine, Royal Melbourne Hospital, Melbourne, Australia; ${ }^{2}$ Department of Medicine (RMH), University of \\ Melbourne, Parkville, Australia; ${ }^{3}$ Department of Respiratory Medicine, Austin Hospital, Heidelberg, Victoria, Australia; ${ }^{4}$ Personalised Oncology \\ Division, Walter Eliza Hall institute, Melbourne, Australia; ${ }^{5}$ School of Health and Biomedical Sciences, RMIT University, Bundoora, Victoria, \\ Australia; ${ }^{6}$ Department of Pathology, Royal Melbourne Hospital, Melbourne, Australia; ${ }^{7}$ Peter MacCallum Cancer Centre, Melbourne, Australia; \\ ${ }^{8}$ Department of Thoracic Surgery, Royal Melbourne Hospital, Melbourne, Australia \\ Contributions: (I) Conception and design: K Rangamuwa, D Steinfort; (II) Administrative support: K Rangamuwa, D Steinfort; (III) Provision of study \\ materials or patients: None; (IV) Collection and assembly of data: None; (V) Data analysis and interpretation: None; (VI) Manuscript writing: All \\ authors; (VII) Final approval of manuscript: All authors. \\ Correspondence to: Dr. Kanishka Rangamuwa. Respiratory Department, Royal Melbourne Hospital, 300 Grattan St, Parkville, Victoria 3050, Australia. \\ Email: kanishka.rangamuwa@mh.org.au.
}

\begin{abstract}
Lung cancer is the leading cause of cancer death worldwide, with approximately 1.6 million cancer related deaths each year. Prognosis is best in patients with early stage disease, though even then five-year survival is only $55 \%$ in some groups. Median survival for advanced non-small cell lung cancer (NSCLC) is 8-12 months with conventional treatment. Immune checkpoint inhibitor (ICI) therapy has revolutionised the treatment of NSCLC with significant long-term improvements in survival demonstrated in some patients with advanced NSCLC. However, only a small proportion of patients respond to ICI, suggesting the need for further techniques to harness the potential of ICI therapy. Thermal ablation utilizes the extremes of temperature to cause tumour destruction. Commonly used modalities are radiofrequency ablation (RFA), cryoablation and microwave ablation (MWA). At present thermal ablation is reserved for curative-intent therapy in patients with localized NSCLC who are unable to undergo surgical resection or stereotactic ablative body radiotherapy (SABR). Limited evidence suggests that thermal ablative modalities can upregulate an anticancer immune response in NSCLC. It is postulated that thermal ablation can increase tumour antigen release, which would initiate and upregulated steps in the cancer immunity cycle required to elicit an anticancer immune response. This article will review the current thermal ablative techniques and their ability to modulate an anti-cancer immune response with a view of using thermal ablation in conjunction with ICI therapy.
\end{abstract}

Keywords: Non-small cell lung cancer (NSCLC); cancer immunity; immunotherapy; thermal ablation

Submitted Sep 26, 2020. Accepted for publication Nov 26, 2020.

doi: $10.21037 /$ tlcr-20-1075

View this article at: http://dx.doi.org/10.21037/tlcr-20-1075

Lung cancer is the leading cause of cancer death worldwide. More than $80 \%$ of lung cancers are non-small cell lung cancer (NSCLC) and patients with early stage disease have the best outcomes. However, most NSCLC are diagnosed at an advanced stage where the median survival is 12 months with conventional treatment of chemotherapy and radiotherapy (1). The recent development of immune checkpoint inhibitor (ICI) therapy (immunotherapy) 
has significantly improved survival in some patients. Unfortunately, not all patients respond to immunotherapy and others who may respond initially can go on to develop resistance $(2,3)$. New approaches are required to fully utilise the benefits of immunotherapy. In this article we review the use of thermal ablation modalities in NSCLC and their potential to enhance natural and immunotherapy antitumour responses.

\section{Thermal ablation modalities}

Thermal ablation uses extreme temperatures to induce tissue damage and is used to treat several malignancies including liver, kidney and lung, as an alternative for curative-intent therapy in medically inoperable patients with Stage I NSCLC $(4,5)$.

The commonly used thermal ablation techniques are radiofrequency ablation (RFA), microwave ablation (MWA) and cryoablation. The mechanism by which each modality induces tumour destruction has been described extensively in the literature (6-9). Ablative modalities are delivered percutaneously directly into the tumour in current clinical practice, but bronchoscopic ablative techniques are in development $(10,11)$.

Cryoablation is the rapid cooling of tissue to a temperature low enough that it results in tissue damage. Cryoablation probes achieve this by harnessing the JouleThompson effect where a drop in temperature occurs as a result for the rapid expansion of certain liquids to gas such as argon or nitrogen. Temperatures as low as $-160^{\circ} \mathrm{C}$ can be achieved in tissue with cryoablation, resulting in ice crystal formation with cell death caused by cell membrane rupture, cell desiccation and osmotic shock (7).

RFA uses the heat energy that is created by high frequency alternating currents to cause tissue damage and coagulative necrosis. Tissue damage is dependent on the electrical conductance of tissue. Low conductance of lung, and close proximity to large blood vessels and airways can reduce the efficacy of RFA $(8,9)$.

MWA uses the heat generated by electromagnetic waves between frequencies of 900-2,500 $\mathrm{MHz}$ to cause cell death. Electromagnetic energy is less dependent on tissue characteristics than RFA and can lead to more accurate and larger ablation zones (6).

\section{Clinical outcomes of thermal ablation}

Thermal ablation is reserved for patients with inoperable localized NSCLC. Overall survival benefit between thermal ablation and stereotactic ablative body radiotherapy (SABR) appears comparable in a few large retrospective studies (12-14). The ability to achieve complete ablation, and the progression free survival with RFA and MWA have been found to be comparable, particularly for those with tumours less than two centimetres in diameter (14-20). Five-year overall survival rates of $27-67 \%$ have been reported with cryoablation, RFA and MWA (21-23). This is comparable to SABR, where five-year survival is approximately $30 \%$ and is inferior to five-year survival rates of those who undergo surgical resection were rates are 48-65\% depending on type of surgery and degree of lymph node involvement (24).

The complications of thermal ablation techniques relate to route of delivery (percutaneous or bronchoscopic) and to consequences of tissue ablation. Pneumothorax rates of $30-40 \%$ are described with the percutaneous approach $(14,23,25,26)$. Of these about $13 \%$ will require chest tube insertion $(14,23,25)$. Complications related to the consequences of tissue ablation include pleural effusion (5.2-9.6\%), haemoptysis (3.9\%), pneumonia (5.7\%), respiratory failure (3.5\%) and lung collapse (4\%) (26). Clinically significant haemorrhage is rare. In the same way that bronchoscopic techniques have proven far safer than percutaneous biopsy for diagnosis of peripheral pulmonary lesions (27), bronchoscopic ablative modalities are associated with a superior safety profile, with initial studies demonstrating minimal adverse events (28-32).

\section{Cancer and the immune system}

The revolutionary improvements in lung cancer treatment with the use of immunotherapy has highlighted the need for a deeper understanding of the role of the immune system in the evolution of cancer $(1,33)$.

Oncogenesis requires multiple events that enable tumour cell survival, including multiple somatic mutations that activate oncogenic drivers or delete tumour suppressors. The accumulation of mutations can result in cancer cells that are genetically diverse from the patient and as such should be identified as foreign by the host immune system, resulting in an anti-cancer immune response.

The cancer-immunity cycle illustrates the steps that are required to enable an anti-cancer immune response (Figure 1). Cancer antigens are presented via antigen presenting cells such as dendritic cells to $\mathrm{T}$ cells causing activation and priming of the immune response. This leads to trafficking and infiltration of cytotoxic $\mathrm{T}$ cells 


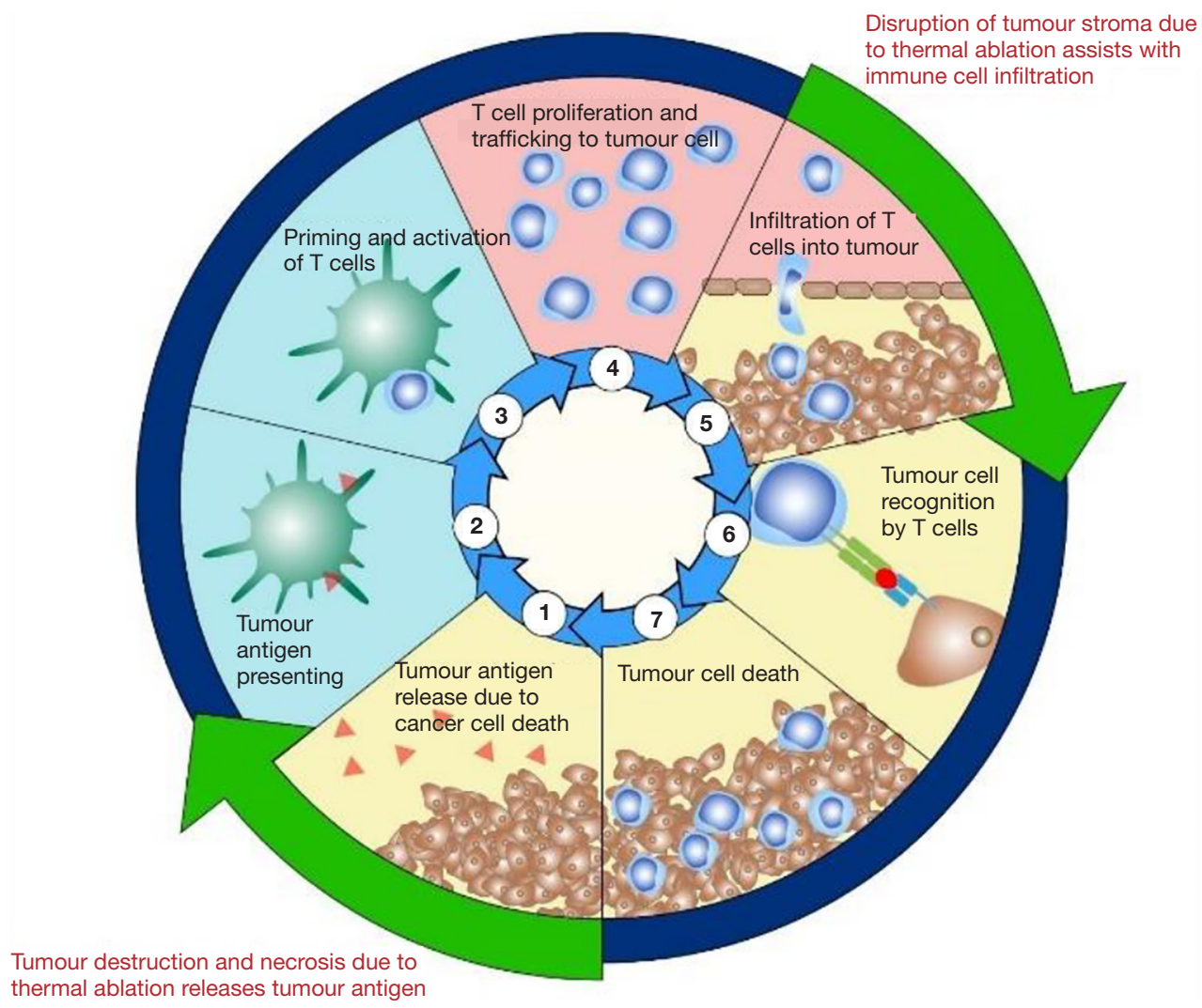

Figure 1 The cancer immunity cycle and the effects of thermal ablation: the cancer immunity cycle demonstrates the steps needed to induce an anticancer immune response. Step 1: tumour antigen release; step 2: Antigen presenting by dendritic cells; step 3: Priming and activation of T cells; step 4: T cell proliferation and trafficking to tumour cells; step 5: infiltration of T cells into tumour; step 6: tumour cell recognition by $\mathrm{T}$ cells and step 7: Tumour cell death. Immune checkpoint inhibitors can assist with steps 3, 6 and 7. Current evidence would suggest that thermal ablation would upregulate steps 1 and 2 as well as augment step 5 .

into the tumour resulting in cancer cell death and further antigen release (34). A hallmark of tumour development is immunoevasion (35), which often occurs through upregulation of negative regulatory pathways (checkpoints) of immune homeostasis. Two examples are cytotoxic T-lymphocyte protein 4 (CTLA-4) and programmed cell death protein 1 (PD-1). CTLA-4 limits T cell activation by directly competing with the ligands of the co-stimulatory protein CD28, CD80 and CD86 (36). PD-1 is a cell surface receptor expressed on T-cells. Binding to its ligand program cell death ligand 1 (PD-L1) (Figure 2), results in activation of inhibitory signal pathways that lead to T-cell attenuation and exhaustion (37-41). Durvalumab, Pembrolizumab and nivolumab are anti-PD-L1 monoclonal antibodies that are currently used in current clinical practise. Ipilimumab is an anti-CTLA-1 monoclonal antibody that has recently been approved for use in NSCLC. However, despite the excellent response to ICI therapy in some patients with durable responses that can last many years, this only appears to occur in about $20 \%$ of those that are treated (2). Some patients with initial respond to PD-1 inhibitors go on to develop resistance as well (3).

Response to ICI therapy appears to correlate with types of T-cell immune response. Several studies that have assessed histologic samples prior to initiation of therapy have demonstrated several immune phenotypes that predict response to immunotherapy. These profiles are immuneinflamed, immune excluded and immune-desert phenotypes. The immune inflamed phenotype is characterised by a tumour microenvironment where immune cells (especially CD4+ and CD8+ $\mathrm{T}$ cells) and cancer cells are in close proximity within the tumour parenchyma. These tumours are also associated with elevated levels of pro-inflammatory cytokines that promote $\mathrm{T}$ cell activation and expansion. 


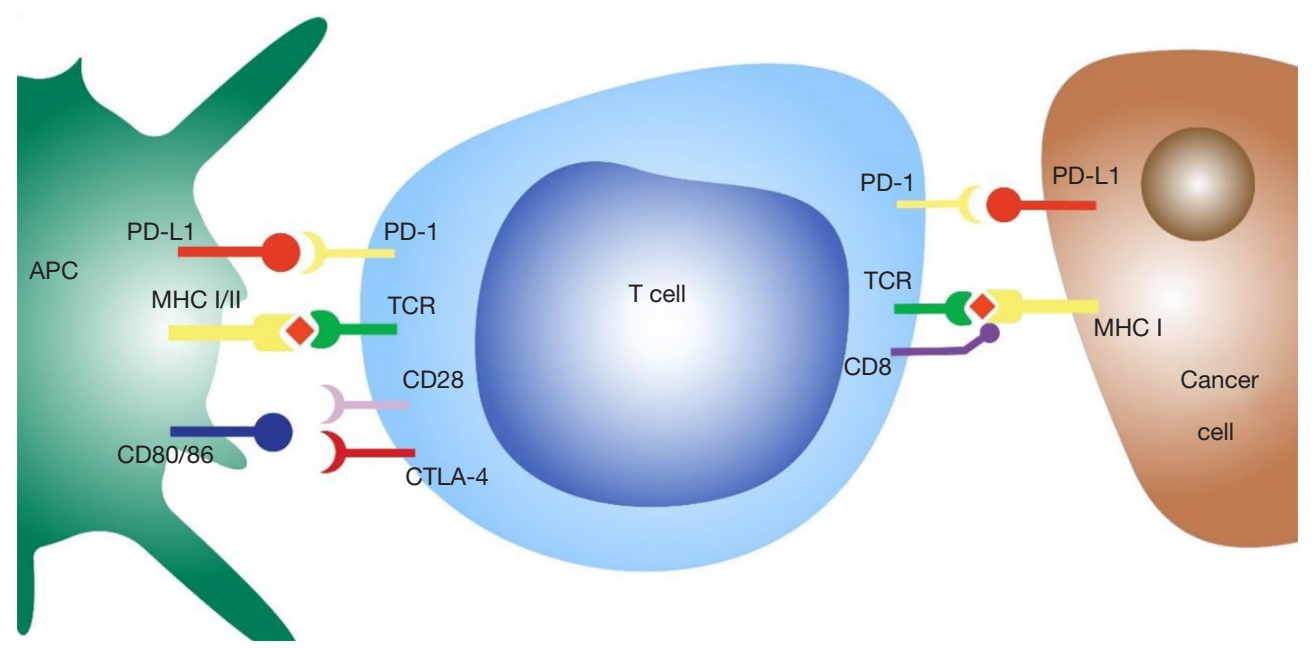

Figure $2 \mathrm{~T}$ cell interaction with antigen presenting cells and tumour cells. APC, antigen presenting cell; PD-1, programmed cell death protein 1; PD-L1, programmed cell death ligand 1; MHC, major histocompatibility complex; CTLA4, cytotoxic T lymphocyte protein 4; TCR, T cell receptor.

The immune-excluded phenotype demonstrates abundant immune cells; however, these cells are found in the stroma and do not penetrate the tumour parenchyma. The immune-desert phenotype shows a paucity of $\mathrm{T}$ cells in both the stroma and parenchyma of the tumour $(42,43)$.

Immune-inflamed tumours have higher response rates to anti-PD-L1 and anti-PD-1 therapy. However, not all immune-inflamed tumours respond to immunotherapy, highlighting that immune cell infiltration on its own is not sufficient to induce an immune response and that implies cancer immunity is affected by tumour, host and environmental factors. These include intrinsic tumour properties such as cytokine release (44) and genetic composition (44) [including tumour mutational burden (TMB)]; and extrinsic factors such as the gut microbiome (45-49), the presence of infection (44) and the exposure to sunlight (50). These factors promote and suppress cancer immunity and sit in an equilibrium that is defined as the cancer-immune set point. This threshold needs to be surpassed for an individual with cancer to respond to immunotherapy (44).

\section{Thermal ablation and the immune system}

Recent pre-clinical work and some clinical studies have suggested that percutaneous thermal ablative therapies may alter the immune-profile of patients with cancer by activating various steps in the cancer immunity cycle. This would imply that thermal ablation has the potential to improve the efficacy of immunotherapy $(51,52)$. We will explore further the current evidence that supports this for RFA, cryoablation and MWA.

Though evidence is limited in NSCLC, immunogenic changes with thermal ablation have been assessed in several malignancies, especially liver cancer, breast cancer, renal cell cancer and prostate cancer. Early studies in both cryoablation and RFA demonstrated tumour regression of untreated metastatic lesions (abscopal effect) with associated upregulation of immune cells in peripheral blood (53-59).

Tumour necrosis occurs due to thermal ablation and results in neoantigen release from the tumour. RFA has been shown to increase carcinoembryonic antigen (CEA) levels in liver metastasis of colorectal cancer and similar changes in serum prostate specific antigen (PSA) in prostate cancer has been seen with cryoablation $(60,61)$. Along with an increase in neoantigen release, an upregulation of danger signals is seen and together this assists in modulating the first step of the cancer immunity cycle. Danger signals are endogenous molecules that are released by damaged cells and have various effects on cancer immunity. Heat shock protein 70 (HSP70) is a danger signal that has been found to be elevated post cryoablation and RFA in melanoma (62). Heat-shock proteins assists in antigen presenting, by both chaperoning antigen to dendritic cells and by upregulating MHC class 1 expression $(63,64)$. This induction of neoantigen expression and danger signal release leads 
to increased neoantigen presentation as evidenced by the increased numbers of dendritic cells and enhanced dendritic cell maturation that has been observed with both cryoablation and RFA in several cancers including NSCLC $(65,66)$.

Evidence of $\mathrm{T}$ cell trafficking to ablated tumour as a result of increased neoantigen presentation has been seen in preclinical studies. Chemokine ligand 21 (CCL21) and intercellular adhesion molecule 1 (ICAM-1) are key molecules associated with $\mathrm{T}$ cell trafficking. The expression of CCL21 and ICAM-1 have been shown to be overexpressed in endothelial venules of the tumour draining lymph node and in CD31 positive endothelial cells of the tumour post RFA. As a result, ablation creates a localised T cell homing function causing an increase in naïve CD8+ T cells in ablated tumour tissue and tumour draining lymph nodes. The increase in $\mathrm{T}$ cell trafficking can be seen as early as 1 hour post ablation in mouse studies and appears to peak somewhere between 6 to 12 hours post ablation (67) leading to an increase in tumour infiltrating lymphocytes.

Preclinical and clinical studies have shown that the lymphocytes that infiltrate the tumour as a result of thermal ablation are predominantly CD4+ and CD $8+\mathrm{T}$ cells (54,56,66,68-77). This response appears more robust with RFA and cryoablation than MWA $(72,73,78)$. Upregulation of PD-L1 expression in resected tumour and PD1 expression in tumour infiltrating CD8+ and CD4+ lymphocytes have been seen post RFA in colorectal cancer (74). Natural killer (NK) cell and macrophage infiltration also appears to increase with thermal ablation $(71,73)$.

This increase in tumour infiltrating lymphocytes has been associated with elevated interferon- $\gamma$ (IFN- $\gamma$ ) levels post ablation, implying an upregulation of a $\mathrm{T}$ helper 1 (Th1) response that in turn would correspond with a cell mediated immune response (79). A Th1 response has also been observed in patients with liver cancer who demonstrate an abscopal effect with thermal ablation. Furthermore, the degree of the Th1 response appears to correlate with the observed clinical response in prostate cancer and renal cell carcinoma $(80,81)$. Additionally, the pro-inflammatory cytokines IL-1 $\beta$, IL- 6 and IL- 8 have been noted to be elevated post thermal ablation $(54,82,83)$. These cytokines have been shown to assist with $\mathrm{T}$ cell proliferation and trafficking (84); as well promoting a Th1 response (85).

Importantly, along with these changes in $\mathrm{T}(\mathrm{CD} 4+$ and CD8+) effector cells, a reduction of immunosuppressive regulatory $\mathrm{T}$ cells (Treg) post ablation has been noted in studies of renal cell cancer, hepatocellular cancer (HCC) and prostate cancer $(54,77,86-88)$. A higher number of CD4+ and CD8+ T cells and a lower number of Treg cells and myeloid-derived suppressor cells (MDSC) post thermal ablation have been shown to have a positive effect on tumour progression and survival $(86,89)$.

While most of the evidence supports a robust anti-cancer immune response with thermal ablation it only seems to be transient. The immune effect appears to last about 4 weeks, as described in a preclinical study where immune cells taken 4 and 8 weeks post ablation underwent a tumour rechallenge and anti-tumour cytolytic effect was only seen with immune cells from 4 weeks post ablation (90). In human studies, tumour antigen specific CD4+ and CD8+ $\mathrm{T}$ cells have been seen to persist in the peripheral blood between 2-4 weeks post ablation $(54,76,77)$.

\section{Thermal ablation and immunotherapy}

While the anti-cancer immune response post ablation appears transient, there is robust evidence that it upregulates various steps in the cancer immunity cycle and could act to enhance the effect of immunotherapies. This has been assessed in some preclinical and clinical studies (Table 1).

Several types of immunotherapy have been assessed in combination with thermal ablation. These include dendritic cell injection; the use of the immune adjuvant CPGoligodeoxynucleotides (CpG-ODN); injection of OK432 activated dendritic cells; IL-2 injection and granulocyte macrophage colony stimulating factor (GM-CSF) injection. All therapies in conjunction with thermal ablation appeared to promote a stronger immune response than either ablation or immunotherapy alone (Table 1) $(62,69,71,74,81,91-110)$.

More importantly, a few studies have combined ICI therapy with thermal ablation in both preclinical and clinical settings. Similar to other immunotherapies combination, the addition of ICI to thermal ablation appeared to produce a more robust response compared to either ICI or ablation alone (Table 1) $(74,92,97,100,105,106,109)$.

One pilot study by McArthur et al. assessed patients with breast cancer who were treated with combined cryoablation and a CTLA-4 antagonist prior to mastectomy. Combination treatment was observed to be safe in this cohort. Combination treatment demonstrated a more sustained immune response than either ICI or thermal ablation alone. In peripheral blood this was characterised by an increase in Th1 cytokines (IFN- $\gamma$ ) and activated (ICOS+) and proliferating (Ki67+) CD4+T 


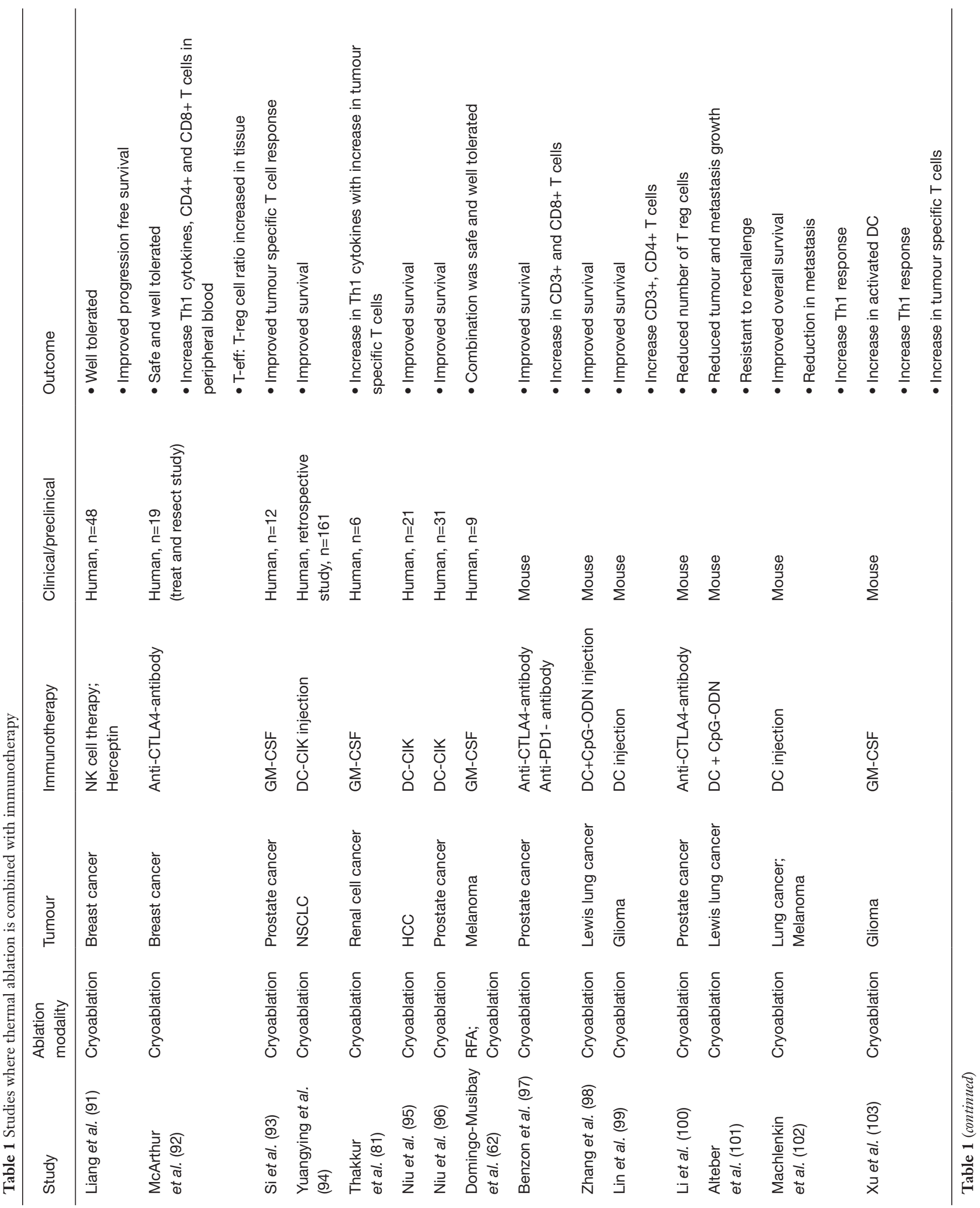




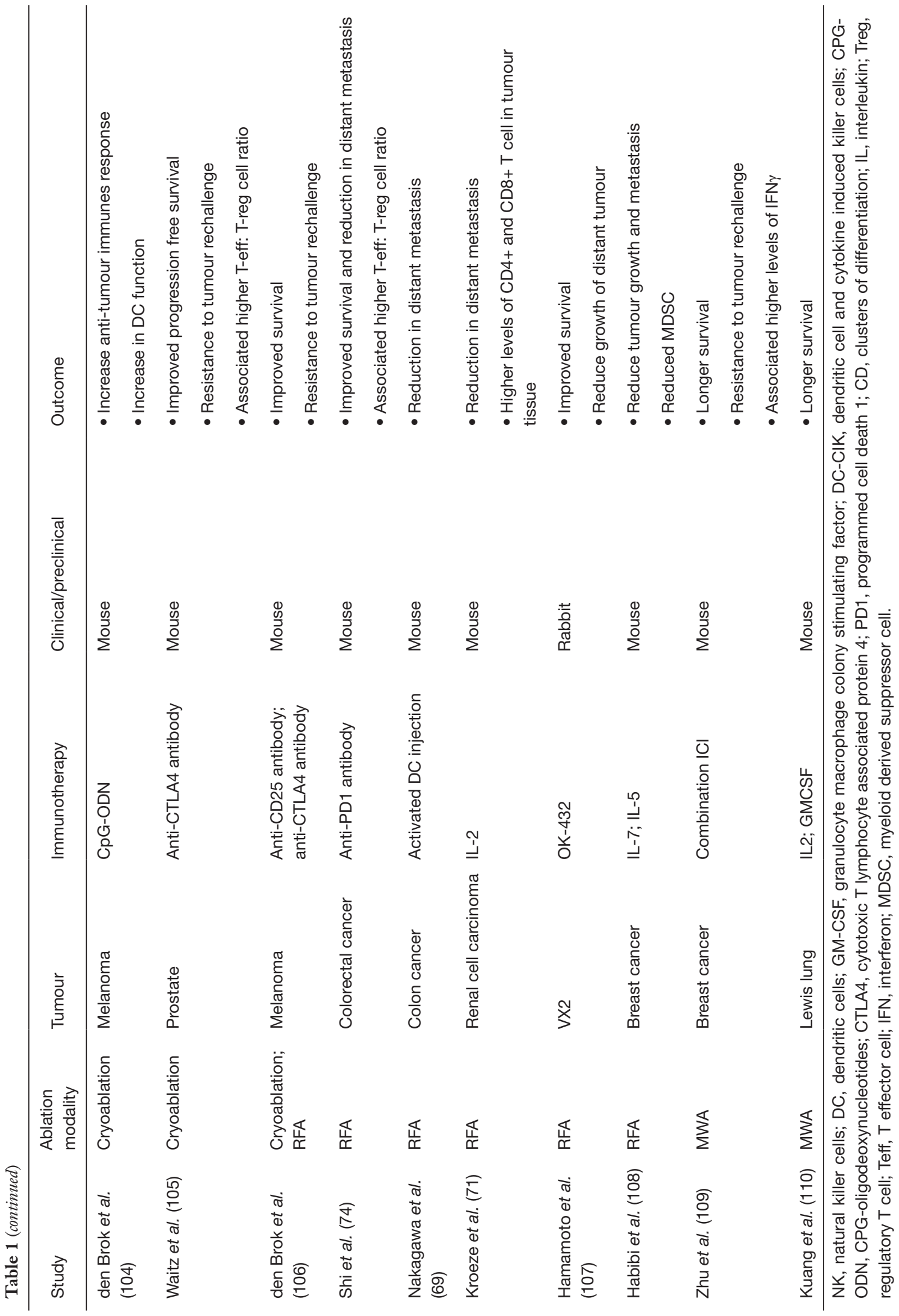


Table 2 Studies combining thermal ablation and immunotherapy that are currently registered at clinicaltrials.gov

\begin{tabular}{llc}
\hline Identifier & Title & Primary endpoint \\
\hline $\begin{array}{l}\text { NCT04339218 } \\
(111)\end{array}$ & $\begin{array}{l}\text { Cryoablation in Combination (or Not) With Pembrolizumab and Pemetrexed-carboplatin in } \\
\text { 1st-line Treatment for Patients with Metastatic Lung Adenocarcinoma (CRYOMUNE) }\end{array}$ & 1-year overall survival \\
$\begin{array}{l}\text { NCT04201990 } \\
(112)\end{array}$ & $\begin{array}{l}\text { Cryoablation Combined with Camrelizumab and Apatinib for Multiprimary Lung Cancer } \\
\text { (CCA-MPLC) }\end{array}$ & Safety \\
NCT04102982 & Microwave Ablation in Combination with Camrelizumab Versus Camrelizumab in Metastatic & Overall survival \\
$(113)$ & Non-small-cell Lung Cancer (MWA in NSCLC) & Overall survival \\
NCT03769129 & Evaluating the Safety and Efficacy of Pembrolizumab Combined with MWA for Advanced & \\
$(114)$ & NSCLC & Study of Core Needle Biopsy and Cryoablation of an Enlarging Tumour in Patients with \\
NCT03290677 & Metastatic Lung Cancer and Metastatic Melanoma Receiving Post-Progression Immune & Cumulative incidence of \\
$(115)$ & Checkpoint Inhibitor Therapy & adverse events \\
\hline
\end{tabular}

cells and CD8+ T cells (92). Currently further trials are underway to assess the synergy of thermal ablation with ICI in NSCLC (111-115) (Table 2).

\section{Non thermal ablation and the immune system}

Irreversible electroporation (IRE) is another ablation technique that exposes cells to electric pulses, which leads to cell wall damage and immunogenic cell death. Though not classified as a thermal ablative technique it has shown upregulation of an immune response in pancreatic cancer. In mouse studies with transplantation of renal carcinoma and pancreatic cancer cell lines, IRE lead to an increase in T-cell infiltration and improvements in progression free survival $(116,117)$. IFN- $\gamma$ increase has also been observed post IRE in rat models with osteosarcoma, this again was associated with an increase in CD4+ T cells in peripheral blood and tumour (118). A study of 34 patients with locally advanced pancreatic cancer that underwent IRE demonstrated similar findings with an increase in CD4+ T cells, CD8+ T cells, NK cells and a reduction in regulatory $\mathrm{T}$ cells. This was associated with an increase in IL2 and a decrease in IL-6 and IL-10 (119). Additionally, IRE in combination with PD1 blockade has been found to increase CD8+ T cell tumour infiltration and improve overall survival in mice with pancreatic ductal adenocarcinoma (120). These results suggest that IRE also has potential to upregulate an anticancer immune response. Currently IRE has shown limited utility when delivered percutaneously to lung cancers (121-123), however flexible IRE catheters have been utilised bronchoscopically for management of airway-based inflammation and may be suitable for bronchoscopic IRE treatment of lung cancer (124).

Tumour antigen release, dendritic cell activation and CD8 + $\mathrm{T}$ cell infiltration has also been described with radiotherapy. Preclinical studies where radiotherapy was combined with CTLA-4 blockade and anti-PD1 antibody demonstrated improvement in tumour control and anti-tumour immune effect (125). While the safety of combination ICI and radiotherapy has been established (125), clinical trials assessing the immune changes associated with radiotherapy are still underway (126). At present there are no studies comparing the immune effects of radiotherapy to thermal ablation in NSCLC.

\section{Could ablation be harmful?}

While a majority of studies demonstrate a positive anticancer response the with thermal ablation, a few studies have suggested that RFA may promote tumour recurrence in HCC. Aggressive outgrowth of residual hepatic micro-metastasis had been seen in the surrounding tissue of patients undergoing RFA of HCC (127). A hypoxic microenvironment that occurs in HCC post RFA has been shown to enhance the invasive, chemo-resistant and metastatic abilities of the tumour cells, these changes had been seen in patients that underwent incomplete ablation (128). Perhaps in contrast to lung cancer, a chronic inflammatory state in HCC patients may favour a tumour microenvironment that would enhance tumour growth, as suggested by the Th2 cell dominance pre and post RFA in hepatocellular carcinoma in patients with 
hepatitis C (129).

The cytokines IL-10 and TGF- $\beta$ have also been noted to be elevated post thermal ablation $(54,70,82)$. IL-10 and TGF- $\beta$ are immunosuppressive cytokines that act to inhibit dendritic cell maturation and block co-stimulatory pathways and upregulate $\mathrm{T}$ reg differentiation $(82,130,131)$. Similarly, IL-6 which assists with T cell trafficking also has negative effects on anticancer immunity. It plays a role in impairing dendritic cell maturation, increasing MDSC proliferation and assisting in tumour cell proliferation $(84,132,133)$.

A retrospective case control study of patients with colorectal cancer and liver metastasis who underwent RFA found that those with incomplete ablation demonstrated significantly shorter time to new metastasis and reduced overall survival when compared to complete ablation (134). Incomplete tumour ablation could alter the balance of apoptotic cell death versus necrotic cell death. Apoptotic cell death has been found to be less immunogenic and may actually inhibit an immune response (135-137).

The effects of residual tumour were further assessed in mice with colon cancer where It was found that when compared to complete RFA, incomplete RFA increased the amount of new metastatic lesions and tumour growth. AntiPD-1 treatment did not alter the rate of tumour growth or degree of new metastasis in this cohort. Incomplete RFA was associated with an increase in M2 phenotype macrophages, lower numbers of CD4, CD8 T cells and a higher number of Tregs. Tumour proliferation appears to be driven by residual tumour and an increase in tumour associated macrophages (TAMs) (134).

While similar effects have not be seen in cryoablation and MWA studies, this warrants further assessment.

\section{The role of ablative immunotherapy}

As mentioned previously percutaneous thermal ablation is currently used to treat localized NSCLC patient's ineligible for surgery. Ablative immunotherapy, where thermal ablation is combined with ICI, could potentially expand this treatment modality to a broader patient cohort, including surgical patients, as well as later stage tumours. While ICI has significantly improved the survival in patients with metastatic NSCLC, utilising thermal ablation could further improve both rates of response and duration of efficacy. In addition, thermal ablation may be helpful in patients that develop resistance to ICI by increasing neoantigen release and upregulating the cancer immunity cycle.

The current treatment of early stage NSCLC is surgical resection, however 5 -year survival is only $55 \%$ in some groups, with a large proportion experiencing disease recurrence (138). The use of neoadjuvant ICI therapy has been encouraging in initial studies with complete pathologic response noted in some cases. However, the efficacy again is still limited and using this in conjunction with thermal ablation could improve efficacy. The utility of neoadjuvant ablative immunotherapy would need to be assessed in robust clinical trials to assess its efficacy when compared to current standard treatment.

Current evidence demonstrates that thermal ablation can elicit a significant immune response that may be able to propagate the cancer immunity cycle. We hypothesise that this can shift tumours with an immune desert or immuneexcluded phenotype to a more immune-inflamed phenotype by upregulating steps 1 and 2 of the cancer immunity cycle (Figure 1). Furthermore, the process of ablation has the ability to directly disrupt the tumour stroma and upregulate immune cell trafficking, which would also assist in shifting tumours to a more immune-inflamed phenotype.

Along with the changes in immune cell populations and their locations within the tumour microenvironment, the cytokine release that occurs with ablation has been shown in most circumstances to create a pro-inflammatory tumour microenvironment, the hope is that these changes can either alter the cancer immune setpoint for these patients, or modify the balance to a more immune stimulatory phase that shifts the patient/tumour over the threshold needed to respond to ICI.

This hypothesis raises the exciting prospect that the potential of ICI therapy could be further enhanced by combination with ablative local therapy. Initial studies in other cancers would suggest that combination treatment can produce a more durable immune response with a reasonable safety profile.

\section{What is the next step?}

At present we still need a more robust assessment of the immune response induced by thermal ablation. Human studies in NSCLC where the tumour microenvironment is assessed pre and post ablation would be particularly powerful. Assessment of immune cell populations and cytokines, their location and function with techniques such as RNA sequencing and mass cytometry and multiplex immunohistochemistry would allow for detailed analysis of changes with ablation (139-141). Investigation of CD8+ and CD4+ T cells would be especially important as the 
presence and activation status of these cells appear to be an indicator of a strong anti-cancer immune response (43). Once a robust immune response has been demonstrated in NSCLC, performing clinical studies demonstrating tolerability/safety of the combination, and improved treatment outcomes will be needed.

The correlation between response to ablative immunotherapy and other suggested biomarkers predictive of response to ICI such as tumour mutational burden (TMB) would also be important. TMB is the total number of nonsynonymous mutations in the coding regions of genes, and recent evidence suggests that this is a powerful biomarker for selecting patients that will respond to immunotherapy (142). At present PD-L1 expression is used clinically to predict response to ICI, though more recent studies have demonstrated that the response to ICI might be independent of PD-1/PD-L1 expression (33). PD-1 and PD-L1 expression can be altered with ablation, and examining the potential effect of ablation on TMB would be of interest. TMB represent intrinsic tumour properties and alteration in TMB would provide insights into the possible changes to the cancer immune set point. Serial assessment of these biomarkers may be undertaken bronchoscopically to evaluate response to thermal ablation or ablative immunotherapy (143). Understanding responses within the innate intrinsic immune system $(144,145)$ and how these impact responses to immunotherapy will also be important in integrating thermal tumour ablation into treatment paradigms.

\section{Conclusions}

ICI therapy has transformed treatment responses in NSCLC, though responses remain limited to a minority of patients. Immunogenic responses to tumour ablation are well established, and emerging evidence suggests that combination tumour ablation and immunotherapy may augment anti-tumour immune responses. Prospective studies are underway examining clinical efficacy of combination therapy, and studies should focus on both neoadjuvant and metastatic disease settings. Development of delivery systems with improved safety profiles is needed. Bronchoscopic thermal ablative techniques, which are currently under development may achieve this.

\section{Acknowledgments}

Funding: None.

\section{Footnote}

Provenance and Peer Review: This article was commissioned by the editorial office, Translational Lung Cancer Research for the series "Lung cancer and the immune system". The article has undergone external peer review.

Conflicts of Interest: All authors have completed the ICMJE uniform disclosure form (available at http://dx.doi. org/10.21037/tlcr-20-1075). The series "Lung cancer and the immune system" was commissioned by the editorial office without any funding or sponsorship. DS served as the unpaid Guest Editor of the series and serves as an unpaid editorial board member of Translational Lung Cancer Research from Sep 2019 to Sep 2021. The authors have no other conflicts of interest to declare.

Ethical Statement: The authors are accountable for all aspects of the work in ensuring that questions related to the accuracy or integrity of any part of the work are appropriately investigated and resolved.

Open Access Statement: This is an Open Access article distributed in accordance with the Creative Commons Attribution-NonCommercial-NoDerivs 4.0 International License (CC BY-NC-ND 4.0), which permits the noncommercial replication and distribution of the article with the strict proviso that no changes or edits are made and the original work is properly cited (including links to both the formal publication through the relevant DOI and the license). See: https://creativecommons.org/licenses/by-nc-nd/4.0/.

\section{References}

1. Reck M, Rabe KF. Precision Diagnosis and Treatment for Advanced Non-Small-Cell Lung Cancer. N Engl J Med 2017;377:849-61.

2. Lievense LA, Sterman DH, Cornelissen R, et al. Checkpoint Blockade in Lung Cancer and Mesothelioma. Am J Respir Crit Care Med 2017;196:274-82.

3. Gettinger SN, Wurtz A, Goldberg SB, et al. Clinical Features and Management of Acquired Resistance to PD-1 Axis Inhibitors in 26 Patients With Advanced Non-Small Cell Lung Cancer. J Thorac Oncol 2018;13:831-9.

4. Postmus PE, Kerr KM, Oudkerk M, et al. Early and locally advanced non-small-cell lung cancer (NSCLC): ESMO Clinical Practice Guidelines for diagnosis, treatment and follow-up. Ann Oncol 2017;28 Suppl 4:iv1-iv21. 
5. Howington JA, Blum MG, Chang AC, et al. Treatment of stage I and II non-small cell lung cancer: Diagnosis and management of lung cancer, 3rd ed: American College of Chest Physicians evidence-based clinical practice guidelines. Chest 2013;143:e278S-e313S.

6. Lubner MG, Brace CL, Hinshaw JL, et al. Microwave tumor ablation: mechanism of action, clinical results, and devices. J Vasc Interv Radiol 2010;21:S192-203.

7. Erinjeri JP, Clark TW. Cryoablation: mechanism of action and devices. J Vasc Interv Radiol 2010;21:S187-91.

8. Hong K, Georgiades C. Radiofrequency ablation: mechanism of action and devices. J Vasc Interv Radiol 2010;21:S179-86.

9. Bargellini I, Bozzi E, Cioni R, et al. Radiofrequency ablation of lung tumours. Insights Imaging 2011;2:567-76.

10. Steinfort DP, Herth FJF. Bronchoscopic treatments for early-stage peripheral lung cancer: Are we ready for prime time? Respirology 2020;25:944-52.

11. Steinfort DP, Christie M, Antippa P, et al. Bronchoscopic Thermal Vapour Ablation for Localized Cancer Lesions of the Lung: A Clinical Feasibility Treat-and-Resect Study. Respiration 2021;100:432-42.

12. Lam A, Yoshida EJ, Bui K, et al. A National Cancer Database Analysis of Radiofrequency Ablation versus Stereotactic Body Radiotherapy in Early-Stage Non-Small Cell Lung Cancer. J Vasc Interv Radiol 2018;29:1211-7.e1.

13. Uhlig J, Ludwig JM, Goldberg SB, et al. Survival Rates after Thermal Ablation versus Stereotactic Radiation Therapy for Stage 1 Non-Small Cell Lung Cancer: A National Cancer Database Study. Radiology 2018;289:862-70.

14. Yuan Z, Wang Y, Zhang J, et al. A Meta-Analysis of Clinical Outcomes After Radiofrequency Ablation and Microwave Ablation for Lung Cancer and Pulmonary Metastases. J Am Coll Radiol 2019;16:302-14.

15. de Baere T, Palussiere J, Auperin A, et al. Midterm local efficacy and survival after radiofrequency ablation of lung tumors with minimum follow-up of 1 year: prospective evaluation. Radiology 2006;240:587-96.

16. Gillams AR, Lees WR. Radiofrequency ablation of lung metastases: factors influencing success. Eur Radiol 2008;18:672-7.

17. Hiraki T, Sakurai J, Tsuda T, et al. Risk factors for local progression after percutaneous radiofrequency ablation of lung tumors: evaluation based on a preliminary review of 342 tumors. Cancer 2006;107:2873-80.

18. Okuma T, Matsuoka T, Yamamoto A, et al. Determinants of local progression after computed tomography-guided percutaneous radiofrequency ablation for unresectable lung tumors: 9-year experience in a single institution. Cardiovasc Intervent Radiol 2010;33:787-93.

19. Lencioni R, Crocetti L, Cioni R, et al. Response to radiofrequency ablation of pulmonary tumours: a prospective, intention-to-treat, multicentre clinical trial (the RAPTURE study). Lancet Oncol 2008;9:621-8.

20. de Baere T, Auperin A, Deschamps F, et al. Radiofrequency ablation is a valid treatment option for lung metastases: experience in 566 patients with 1037 metastases. Ann Oncol 2015;26:987-91.

21. Simon CJ, Dupuy DE, DiPetrillo TA, et al. Pulmonary radiofrequency ablation: long-term safety and efficacy in 153 patients. Radiology 2007;243:268-75.

22. Lam A, Yoshida EJ, Bui K, et al. Patient and Facility Demographics Related Outcomes in Early-Stage NonSmall Cell Lung Cancer Treated with Radiofrequency Ablation: A National Cancer Database Analysis. J Vasc Interv Radiol 2018;29:1535-41.e2.

23. Moore W, Talati R, Bhattacharji P, et al. Five-year survival after cryoablation of stage I non-small cell lung cancer in medically inoperable patients. J Vasc Interv Radiol 2015;26:312-9.

24. Chi A, Fang W, Sun Y, et al. Comparison of Longterm Survival of Patients With Early-Stage Non-Small Cell Lung Cancer After Surgery vs Stereotactic Body Radiotherapy. JAMA Network Open 2019;2:e1915724.

25. Lyons GR, Askin G, Pua BB. Clinical Outcomes after Pulmonary Cryoablation with the Use of a Triple Freeze Protocol. J Vasc Interv Radiol 2018;29:714-21.

26. Welch BT, Brinjikji W, Schmit GD, et al. A national analysis of the complications, cost, and mortality of percutaneous lung ablation. J Vasc Interv Radiol 2015;26:787-91.

27. Steinfort DP, Vincent J, Heinze S, et al. Comparative effectiveness of radial probe endobronchial ultrasound versus CT-guided needle biopsy for evaluation of peripheral pulmonary lesions: a randomized pragmatic trial. Respir Med 2011;105:1704-11.

28. Xie F, Zheng X, Xiao B, et al. Navigation Bronchoscopy-Guided Radiofrequency Ablation for Nonsurgical Peripheral Pulmonary Tumors. Respiration 2017;94:293-8.

29. Tsushima K, Koizumi T, Tanabe T, et al. Bronchoscopyguided radiofrequency ablation as a potential novel therapeutic tool. Eur Respir J 2007;29:1193-200.

30. Tanabe T, Koizumi T, Tsushima K, et al. Comparative study of three different catheters for CT imaging- 
bronchoscopy-guided radiofrequency ablation as a potential and novel interventional therapy for lung cancer. Chest 2010;137:890-7.

31. Koizumi T, Kobayashi T, Tanabe T, et al. Clinical experience of bronchoscopy-guided radiofrequency ablation for peripheral-type lung cancer. Case Rep Oncol Med 2013;2013:515160.

32. Koizumi T, Tsushima K, Tanabe T, et al. BronchoscopyGuided Cooled Radiofrequency Ablation as a Novel Intervention Therapy for Peripheral Lung Cancer. Respiration 2015;90:47-55.

33. Antonia SJ, Villegas A, Daniel D, et al. Durvalumab after Chemoradiotherapy in Stage III Non-Small-Cell Lung Cancer. N Engl J Med 2017;377:1919-29.

34. Chen DS, Mellman I. Oncology meets immunology: the cancer-immunity cycle. Immunity 2013;39:1-10.

35. Hanahan D, Weinberg RA. Hallmarks of cancer: the next generation. Cell 2011;144:646-74.

36. Van Coillie S, Wiernicki B, Xu J. Molecular and Cellular Functions of CTLA-4. In: Xu J, editor. Regulation of Cancer Immune Checkpoints: Molecular and Cellular Mechanisms and Therapy. Singapore: Springer Singapore, 2020:7-32.

37. Topalian SL, Hodi FS, Brahmer JR, et al. Safety, activity, and immune correlates of anti-PD-1 antibody in cancer. $\mathrm{N}$ Engl J Med 2012;366:2443-54.

38. Shankaran V, Ikeda H, Bruce AT, et al. IFNgamma and lymphocytes prevent primary tumour development and shape tumour immunogenicity. Nature 2001;410:1107-11.

39. Schreiber RD, Old LJ, Smyth MJ. Cancer immunoediting: integrating immunity's roles in cancer suppression and promotion. Science 2011;331:1565-70.

40. Galon J, Costes A, Sanchez-Cabo F, et al. Type, density, and location of immune cells within human colorectal tumors predict clinical outcome. Science 2006;313:1960-4.

41. Hodi FS, O'Day SJ, McDermott DF, et al. Improved survival with ipilimumab in patients with metastatic melanoma. N Engl J Med 2010;363:711-23.

42. Herbst RS, Soria JC, Kowanetz M, et al. Predictive correlates of response to the anti-PD-L1 antibody MPDL3280A in cancer patients. Nature 2014;515:563-7.

43. Galon J, Bruni D. Approaches to treat immune hot, altered and cold tumours with combination immunotherapies. Nat Rev Drug Discov 2019;18:197-218.

44. Chen DS, Mellman I. Elements of cancer immunity and the cancer-immune set point. Nature 2017;541:321-30.

45. Viaud S, Saccheri F, Mignot G, et al. The intestinal microbiota modulates the anticancer immune effects of cyclophosphamide. Science 2013;342:971-6.

46. Vetizou M, Pitt JM, Daillere R, et al. Anticancer immunotherapy by CTLA-4 blockade relies on the gut microbiota. Science 2015;350:1079-84

47. Sivan A, Corrales L, Hubert N, et al. Commensal Bifidobacterium promotes antitumor immunity and facilitates anti-PD-L1 efficacy. Science 2015;350:1084-9.

48. Iida N, Dzutsev A, Stewart CA, et al. Commensal bacteria control cancer response to therapy by modulating the tumor microenvironment. Science 2013;342:967-70.

49. Garrett WS. Cancer and the microbiota. Science 2015;348:80-6.

50. Dopico XC, Evangelou M, Ferreira RC, et al. Widespread seasonal gene expression reveals annual differences in human immunity and physiology. Nat Commun 2015;6:7000.

51. Takaki H, Cornelis F, Kako Y, et al. Thermal ablation and immunomodulation: From preclinical experiments to clinical trials. Diagn Interv Imaging 2017;98:651-9.

52. Katzman D, Wu S, Sterman DH. Immunological Aspects of Cryoablation of Non-Small Cell Lung Cancer: A Comprehensive Review. J Thorac Oncol 2018;13:624-35.

53. Zerbini A, Pilli M, Penna A, et al. Radiofrequency thermal ablation of hepatocellular carcinoma liver nodules can activate and enhance tumor-specific T-cell responses. Cancer Res 2006;66:1139-46.

54. Fietta AM, Morosini M, Passadore I, et al. Systemic inflammatory response and downmodulation of peripheral CD25+Foxp3+ T-regulatory cells in patients undergoing radiofrequency thermal ablation for lung cancer. Hum Immunol 2009;70:477-86.

55. Hansler J, Wissniowski TT, Schuppan D, et al. Activation and dramatically increased cytolytic activity of tumor specific $\mathrm{T}$ lymphocytes after radio-frequency ablation in patients with hepatocellular carcinoma and colorectal liver metastases. World J Gastroenterol 2006;12:3716-21.

56. Ito $\mathrm{F}, \mathrm{Ku} \mathrm{AW}$, Bucsek MJ, et al. Immune Adjuvant Activity of Pre-Resectional Radiofrequency Ablation Protects against Local and Systemic Recurrence in Aggressive Murine Colorectal Cancer. PLoS One 2015;10:e0143370.

57. Ablin RJ, Guinan PD, Bruns GR, et al. Evaluation of cellular immunologic responsiveness in the clinical management of patients with prostatic cancer. II. Effect of oestrogen, cryosurgery and transurethral resection on thymic-dependent lymphocytic blastogenesis. Urol Int 1976;31:383-400.

58. Airoldi M, Gandolfo S, Salassa B, et al. Evaluation of some nonspecific immunological parameters in patients with 
oral cavity carcinoma undergoing cryosurgery. Boll Soc Ital Biol Sper 1980;56:1334-40.

59. Weyer U, Petersen I, Ehrke C, et al. Immunomodulation by cryosurgery in malignant melanoma. Onkologie 1989;12:291-6.

60. Ghanamah M, Berber E, Siperstein A. Pattern of carcinoembryonic antigen drop after laparoscopic radiofrequency ablation of liver metastasis from colorectal carcinoma. Cancer 2006;107:149-53.

61. Leibovici D, Zisman A, Lindner A, et al. PSA elevation during prostate cryosurgery and subsequent decline. Urol Oncol 2005;23:8-11.

62. Domingo-Musibay E, Heun JM, Nevala WK, et al. Endogenous Heat-Shock Protein Induction with or Without Radiofrequency Ablation or Cryoablation in Patients with Stage IV Melanoma. Oncologist 2017;22:1026-e93.

63. Srivastava P. Interaction of heat shock proteins with peptides and antigen presenting cells: chaperoning of the innate and adaptive immune responses. Annu Rev Immunol 2002;20:395-425.

64. Wells AD, Rai SK, Salvato MS, et al. Hsp72-mediated augmentation of MHC class I surface expression and endogenous antigen presentation. Int Immunol 1998;10:609-17.

65. Yin Z, Lu G, Xiao Z, et al. Antitumor efficacy of argonhelium cryoablation-generated dendritic cell vaccine in glioma. Neuroreport 2014;25:199-204.

66. Schneider T, Hoffmann H, Dienemann H, et al. Immune Response After Radiofrequency Ablation and Surgical Resection in Nonsmall Cell Lung Cancer. Semin Thorac Cardiovasc Surg 2016;28:585-92.

67. Ito F, Vardam TD, Appenheimer MM, et al. In situ thermal ablation augments antitumor efficacy of adoptive T cell therapy. Int J Hyperthermia 2019;36:22-36.

68. Kim HK, Pyun JH, Cho S, et al. Tumor-specific immunity induced by cryoablation in a murine renal cell carcinoma model. Korean J Urol 2014;55:834-40.

69. Nakagawa H, Mizukoshi E, Iida N, et al. In vivo immunological antitumor effect of OK-432-stimulated dendritic cell transfer after radiofrequency ablation. Cancer Immunol Immunother 2014;63:347-56.

70. Erös de Bethlenfalva-Hora C, Mertens JC, et al. Radiofrequency ablation suppresses distant tumour growth in a novel rat model of multifocal hepatocellular carcinoma. Clin Sci (Lond) 2014;126:243-52.

71. Kroeze SG, Daenen LG, Nijkamp MW, et al. Radio frequency ablation combined with interleukin-2 induces an antitumor immune response to renal cell carcinoma in a murine model. J Urol 2012;188:607-14.

72. Zhang H, Hou X, Cai H, et al. Effects of microwave ablation on T-cell subsets and cytokines of patients with hepatocellular carcinoma. Minim Invasive Ther Allied Technol 2017;26:207-11.

73. Zhang J, Dong B, Liang P, et al. Significance of changes in local immunity in patients with hepatocellular carcinoma after percutaneous microwave coagulation therapy. Chin Med J (Engl) 2002;115:1367-71.

74. Shi L, Chen L, Wu C, et al. PD-1 Blockade Boosts Radiofrequency Ablation-Elicited Adaptive Immune Responses against Tumor. Clin Cancer Res2016;22:117384.

75. Nobuoka D, Motomura Y, Shirakawa H, et al. Radiofrequency ablation for hepatocellular carcinoma induces glypican-3 peptide-specific cytotoxic $\mathrm{T}$ lymphocytes. Int J Oncol 2012;40:63-70.

76. Widenmeyer M, Shebzukhov Y, Haen SP, et al. Analysis of tumor antigen-specific $T$ cells and antibodies in cancer patients treated with radiofrequency ablation. J Cancer Res Ther r 2011;128:2653-62.

77. Shaobin W, Yu X, Jiatian L, et al. Changes of CD4+ T-cell subsets after radiofrequency ablation in lung cancer and its significance. J Cancer Ther Res 2016;12:C166-C70.

78. Zhou Y, Xu X, Ding J, et al. Dynamic changes of T-cell subsets and their relation with tumor recurrence after microwave ablation in patients with hepatocellular carcinoma. J Cancer Res Ther 2018;14:40-5.

79. Yang X, Li X, Guo Z, et al. Immunological response induced by cryoablation against murine $\mathrm{H} 22$ hepatoma cell line in vivo. Cryobiology 2018;80:114-8.

80. Osada S, Imai H, Yawata K, et al. Growth inhibition of unresectable tumors induced by hepatic cryoablation: report of two cases. Hepatogastroenterology 2008;55:231-4.

81. Thakur A, Littrup P, Paul EN, et al. Induction of specific cellular and humoral responses against renal cell carcinoma after combination therapy with cryoablation and granulocyte-macrophage colony stimulating factor: a pilot study. J Immunother 2011;34:457-67.

82. Erinjeri JP, Thomas CT, Samoilia A, et al. Image-guided thermal ablation of tumors increases the plasma level of interleukin-6 and interleukin-10. J Vasc Interv Radiol 2013;24:1105-12.

83. Ahmad F, Gravante G, Bhardwaj N, et al. Changes in interleukin- $1 \beta$ and 6 after hepatic microwave tissue ablation compared with radiofrequency, cryotherapy and 
surgical resections. Am J Surg 2010;200:500-6.

84. Fisher DT, Appenheimer MM, Evans SS. The two faces of IL-6 in the tumor microenvironment. Semin Immunol 2014;26:38-47.

85. Knutson KL, Disis ML. Tumor antigen-specific $T$ helper cells in cancer immunity and immunotherapy. Cancer Immunol Immunother 2005;54:721-8.

86. Zhou L, Fu JL, Lu YY, et al. Regulatory T cells are associated with post-cryoablation prognosis in patients with hepatitis B virus-related hepatocellular carcinoma. J Gastroenterol 2010;45:968-78.

87. Si TG, Wang JP, Guo Z. Analysis of circulating regulatory T cells (CD4+CD25+CD127-) after cryosurgery in prostate cancer. Asian J Androl 2013;15:461-5.

88. Li Y, Guo Z, Liu CF, et al. Effect of transcatheter renal arterial embolization combined with cryoablation on regulatory CD4+CD25+ T lymphocytes in the peripheral blood of patients with advanced renal carcinoma. Cryobiology 2012;65:56-9.

89. Mizukoshi E, Yamashita T, Arai K, et al. Enhancement of tumor-associated antigen-specific $\mathrm{T}$ cell responses by radiofrequency ablation of hepatocellular carcinoma. Hepatology 2013;57:1448-57.

90. Si T, Guo Z, Hao X. Immunologic response to primary cryoablation of high-risk prostate cancer. Cryobiology 2008;57:66-71.

91. Liang S, Niu L, Xu K, et al. Tumor cryoablation in combination with natural killer cells therapy and Herceptin in patients with HER2-overexpressing recurrent breast cancer. Mol Immunol 2017;92:45-53.

92. McArthur HL, Diab A, Page DB, et al. A Pilot Study of Preoperative Single-Dose Ipilimumab and/or Cryoablation in Women with Early-Stage Breast Cancer with Comprehensive Immune Profiling. Clin Cancer Res 2016;22:5729-37.

93. Si T, Guo Z, Hao X. Combined cryoablation and GMCSF treatment for metastatic hormone refractory prostate cancer. J Immunother 2009;32:86-91.

94. Yuanying Y, Lizhi N, Feng M, et al. Therapeutic outcomes of combining cryotherapy, chemotherapy and DC-CIK immunotherapy in the treatment of metastatic non-small cell lung cancer. Cryobiology 2013;67:235-40.

95. Niu LZ, Li JL, Zeng JY, et al. Combination treatment with comprehensive cryoablation and immunotherapy in metastatic hepatocellular cancer. World J Gastroenterol 2013;19:3473-80.

96. Niu L, Chen J, He L, et al. Combination treatment with comprehensive cryoablation and immunotherapy in metastatic pancreatic cancer. Pancreas 2013;42:1143-9.

97. Benzon B, Glavaris SA, Simons BW, et al. Combining immune check-point blockade and cryoablation in an immunocompetent hormone sensitive murine model of prostate cancer. Prostate Cancer Prostatic Dis 2018;21:126-36.

98. Zhang M, Yin T, Lu Y, et al. The Application of Cytidyl Guanosyl Oligodeoxynucleotide Can Affect the Antitumor Immune Response Induced by a Combined Protocol of Cryoablation and Dendritic Cells in Lewis Lung Cancer Model. Med Sci Monit 2016;22:1309-17.

99. Lin C, Wang Q, Lu G, et al. In-situ administration of dendritic cells following argon-helium cryosurgery enhances specific antiglioma immunity in mice. Neuroreport 2014;25:900-8.

100.Li F, Guo Z, Yu H, et al. Anti-tumor immunological response induced by cryoablation and anti-CTLA-4 antibody in an in vivo RM-1 cell prostate cancer murine model. Neoplasma 2014;61:659-71.

101.Alteber Z, Azulay M, Cafri G, et al. Cryoimmunotherapy with local co-administration of ex vivo generated dendritic cells and $\mathrm{CpG}-\mathrm{ODN}$ immune adjuvant, elicits a specific antitumor immunity. Cancer Immunol Immunother 2014;63:369-80.

102.Machlenkin A, Goldberger O, Tirosh B, et al. Combined dendritic cell cryotherapy of tumor induces systemic antimetastatic immunity. Clin Cancer Res 2005;11:4955-61.

103.Xu H, Wang Q, Lin C, et al. Synergism between cryoablation and GM-CSF: enhanced immune function of splenic dendritic cells in mice with glioma. Neuroreport 2015;26:346-53.

104. den Brok MH, Sutmuller RP, Nierkens S, et al. Synergy between in situ cryoablation and TLR9 stimulation results in a highly effective in vivo dendritic cell vaccine. Cancer Res 2006;66:7285-92.

105. Waitz R, Solomon SB, Petre EN, et al. Potent induction of tumor immunity by combining tumor cryoablation with anti-CTLA-4 therapy. Cancer Res 2012;72:430-9.

106. den Brok MH, Sutmuller RP, van der Voort R, et al. In situ tumor ablation creates an antigen source for the generation of antitumor immunity. Cancer Res 2004;64:4024-9.

107. Hamamoto S, Okuma T, Yamamoto A, et al. Radiofrequency ablation and immunostimulant OK432: combination therapy enhances systemic antitumor immunity for treatment of VX2 lung tumors in rabbits. Radiology 2013;267:405-13.

108.Habibi M, Kmieciak M, Graham L, et al. Radiofrequency 
thermal ablation of breast tumors combined with intralesional administration of IL-7 and IL-15 augments anti-tumor immune responses and inhibits tumor development and metastasis. Breast Cancer Res Treat2009; 114:423-31.

109.Zhu J, Yu M, Chen L, et al. Enhanced antitumor efficacy through microwave ablation in combination with immune checkpoints blockade in breast cancer: A pre-clinical study in a murine model. Diagn Interv Imaging 2018;99:135-42.

110.Kuang M, Liu SQ, Saijo K, et al. Microwave tumour coagulation plus in situ treatment with cytokinemicroparticles: induction of potent anti-residual tumour immunity. Int J Hyperthermia 2005;21:247-57.

111. Cryoablation in Combination (or Not) With Pembrolizumab and Pemetrexed-carboplatin in 1stline Treatment for Patients With Metastatic Lung Adenocarcinoma. Available online: https://ClinicalTrials. gov/show/NCT04339218

112. Cryoablation Combined With Camrelizumab and Apatinib for Multiprimary Lung Cancer. Available online: https:// ClinicalTrials.gov/show/NCT04201990

113. Microwave Ablation in Combination With Camrelizumab Versus Camrelizumab in Metastatic Non-small-cell Lung Cancer. Available online: https:/ClinicalTrials.gov/show/ NCT04102982

114. Evaluating the Safety and Efficacy of Pembrolizumab Combined With MWA for Advanced NSCLC. Available online: https://ClinicalTrials.gov/show/NCT03769129

115.Study of Core Needle Biopsy and Cryoablation of an Enlarging Tumor in Patients With Metastatic Lung Cancer and Metastatic Melanoma Receiving Postprogression Immune Checkpoint Inhibitor Therapy. Available online: https://ClinicalTrials.gov/show/ NCT03290677

116. Neal RE 2nd, Rossmeisl JH Jr, Robertson JL, et al. Improved local and systemic anti-tumor efficacy for irreversible electroporation in immunocompetent versus immunodeficient mice. PLoS One 2013;8:e64559.

117. White SB, Zhang Z, Chen J, et al. Early Immunologic Response of Irreversible Electroporation versus Cryoablation in a Rodent Model of Pancreatic Cancer. J Vasc Interv Radiol 2018;29:1764-9.

118. Li X, Xu K, Li W, et al. Immunologic response to tumor ablation with irreversible electroporation. PLoS One 2012;7:e48749.

119. He C, Wang J, Sun S, et al. Immunomodulatory Effect after Irreversible Electroporation in Patients with Locally Advanced Pancreatic Cancer. J Oncol2019;2019:9346017.
120.Zhao J, Wen X, Tian L, et al. Irreversible electroporation reverses resistance to immune checkpoint blockade in pancreatic cancer. Nat Commun 2019;10:899.

121. Dupuy DE, Aswad B, Ng T. Irreversible electroporation in a Swine lung model. Cardiovasc Intervent Radiol 2011;34:391-5.

122. Deodhar A, Dickfeld T, Single GW, et al. Irreversible electroporation near the heart: ventricular arrhythmias can be prevented with ECG synchronization. AJR Am J Roentgenol 2011;196:W330-5.

123.Ricke J, Jürgens JH, Deschamps F, et al. Irreversible electroporation (IRE) fails to demonstrate efficacy in a prospective multicenter phase II trial on lung malignancies: the ALICE trial. Cardiovasc Intervent Radiol 2015;38:401-8.

124. Valipour A, Fernandez-Bussy S, Ing AJ, et al. Bronchial Rheoplasty for Treatment of Chronic Bronchitis. TwelveMonth Results from a Multicenter Clinical Trial. Am J Respir Crit Care Med 2020;202:681-9.

125.Xing D, Siva S, Hanna GG. The Abscopal Effect of Stereotactic Radiotherapy and Immunotherapy: Fool's Gold or El Dorado? Clin Oncol (R Coll Radiol) 2019;31:432-43.

126. Safi S, Beckhove P, Warth A, et al. A randomized phase II study of radiation induced immune boost in operable nonsmall cell lung cancer (RadImmune trial). BMC Cancer 2015;15:988.

127. Nijkamp MW, van der Bilt JDW, de Bruijn MT, et al. Accelerated perinecrotic outgrowth of colorectal liver metastases following radiofrequency ablation is a hypoxiadriven phenomenon. Ann Surg 2009;249:814-23.

128. Tong Y, Yang H, Xu X, et al. Effect of a hypoxic microenvironment after radiofrequency ablation on residual hepatocellular cell migration and invasion. Cancer Sci 2017;108:753-62.

129. Kogame M, Nagai H, Shinohara M, et al. Th2 Dominance Might Induce Carcinogenesis in Patients with HCVrelated Liver Cirrhosis. Anticancer Res 2016;36:4529-36.

130. Taylor A, Verhagen J, Blaser K, et al. Mechanisms of immune suppression by interleukin-10 and transforming growth factor-beta: the role of $\mathrm{T}$ regulatory cells. Immunology 2006;117:433-42.

131.Fu S, Zhang N, Yopp AC, et al. TGF-beta induces Foxp3 + T-regulatory cells from CD4 + CD25 - precursors. Am J Transplant 2004; 4:1614-27.

132. Chomarat P, Banchereau J, Davoust J, et al. IL-6 switches the differentiation of monocytes from dendritic cells to macrophages. Nat Immunol 2000;1:510-4. 
133. Mantovani A, Sica A, Allavena P, et al. Tumor-associated macrophages and the related myeloid-derived suppressor cells as a paradigm of the diversity of macrophage activation. Hum Immunol 2009;70:325-30.

134. Shi L, Wang J, Ding N, et al. Inflammation induced by incomplete radiofrequency ablation accelerates tumor progression and hinders PD-1 immunotherapy. Nat Commun 2019;10:5421.

135. Sidana A. Cancer immunotherapy using tumor cryoablation. Immunotherapy 2014;6:85-93.

136. Viorritto ICB, Nikolov NP, Siegel RM. Autoimmunity versus tolerance: can dying cells tip the balance? Clin Immunol 2007;122:125-34.

137.Peng Y, Martin DA, Kenkel J, et al. Innate and adaptive immune response to apoptotic cells. J Autoimmun 2007;29:303-9.

138. Su S, Scott WJ, Allen MS, et al. Patterns of survival and recurrence after surgical treatment of early stage non-small cell lung carcinoma in the ACOSOG Z0030 (ALLIANCE) trial. J Thorac Cardiovasc Surg 2014;147:747-52: discussion 52-3.

139. Kim N, Kim HK, Lee K, et al. Single-cell RNA sequencing demonstrates the molecular and cellular reprogramming of metastatic lung adenocarcinoma. Nat

Cite this article as: Rangamuwa $\mathrm{K}$, Leong T, Weeden C, Asselin-Labat ML, Bozinovski S, Christie M, John T, Antippa P, Irving L, Steinfort D. Thermal ablation in non-small cell lung cancer: a review of treatment modalities and the evidence for combination with immune checkpoint inhibitors. Transl Lung Cancer Res 2021;10(6):2842-2857. doi: 10.21037/tlcr-20-1075
Commun 2020;11:2285.

140.Hofman P, Badoual C, Henderson F, et al. Multiplexed Immunohistochemistry for Molecular and Immune Profiling in Lung Cancer-Just About Ready for PrimeTime? Cancers 2019;11:27.

141.Hanidziar D, Nakahori Y, Cahill LA, et al. Characterization of pulmonary immune responses to hyperoxia by high-dimensional mass cytometry analyses. Sci Rep 2020;10:4677.

142. Berland L, Heeke S, Humbert O, et al. Current views on tumor mutational burden in patients with non-small cell lung cancer treated by immune checkpoint inhibitors. J Thorac Dis 2019;11:S71-S80.

143. Bozinovski S, Vannitamby A, Rangamuwa K, et al. Integrating endobronchial ultrasound bronchoscopy with molecular testing of immunotherapy biomarkers in non-small cell lung cancer. Transl Lung Cancer Res 2021;10:2779- 87.

144. Aloe C, Wang H, Vlahos R, eta 1. Emerging and multifaceted role of neutrophils in lung cancer. Transl Lung Cancer Res 2021;10:2806-18.

145.Hess JB, Sutherland KD, Best SA. Exploring natural killer cell immunology as a therapeutic strategy in lung cancer. Transl Lung Cancer Res 2021;10:2788-805. 